\title{
Inhibitory Effect of Phytic Acid on LPS Induced BV-2 Microglia Activation
}

\author{
Jinhui Zuo ${ }^{1}$, Fuping Guan ${ }^{3}$, Xin Li², Xiuli Wang ${ }^{2}$, Fengyu Pan ${ }^{1}$, Zheng \\ Zhang $^{1}$, Chao Song ${ }^{1}$
}

\author{
${ }^{1}$ Department of Biochemistry and Molecular Biology, Medical College of Qingdao University, Qingdao,China, \\ 266021 \\ ${ }^{2}$ Experiment Center for Cell and Molecular Biology, Medical College of Qingdao University, Qingdao,China, \\ 266021 \\ ${ }^{3}$ Internal Medicine, Jining Municipal Hospital, Jining, China, 272000
}

\begin{abstract}
Hyperactivation of microglia is the important pathology of neurodegenerative diseases. Being naturally occurring compound, phytic acid (PA) has been found to exert anti-inflammatory effect in MPTP-induced parkinson's disease model of mice, but mechanism needed to be further investigated. In our study, BV-2 microglia were cultured and induced by lipopolysaccharide (LPS) to mimic inflammation models to testify the phytic acid's effect on neuroinflammation. Methods: BV-2 microglia were cultured in vitro. After pretreatment with different doses of PA, lipopolysaccharide (LPS) was added to induce the activation of BV-2 microglia. The cell survival rate was detected by MTT colorimetry, the NO release of microglia was measured by Griess assay, and the concentration of IL-6 in supernatant of cell culture was detected by ELISA assay. DAPI staining was used to detect the changes of apoptosis of activated BV-2 microglia before and after phytic acid treatment. Results: Phytic acid could inhibit the production of NO and IL-6 in BV-2 microglia activated by LPS, and block the excessive activation of BV-2 microglia stimulated by LPS. At the same time, it can promote the apoptosis of over-activated microglia. Therefore, phytic acid may play a potential role on prevention and treatment of neurodegenerative diseases associated with its inhibition on microglial activation.
\end{abstract}

Keywords: Phytic Acid, Lipopolysaccharide, Microglia, Neuroinflammation

\begin{abstract}
Introduction
As important immune effector cells in the brain, microglia play a key role in many neuropathic diseases, such as neurodegenerative diseases, brain trauma and central infectious diseases ${ }^{[1]}$. Under normal conditions, microglia are in a resting state, and pathological stimulation can release oxygen free radicals, inflammatory mediators and cytokines, which can induce inflammatory reaction in the central nervous system and play a defensive and repair role. However, excessive activation can also lead to the release of proinflammatory factors, including IL-1 $\beta$ and IL-6, TNF- $\alpha$, resulting in toxicity to neurons and glial cells, and then damage to the central nervous system ${ }^{[2]}$. Therefore, inhibiting the excessive activation of microglia and alleviating the damage caused by inflammatory reaction is particularly critical for the treatment and prevention of many neurodegenerative diseases $^{[3]}$. Lipopolysaccharide (LPS) is the main component of endotoxin in Gram-negative bacteria. It can induce excessive activation of BV-2 microglia and release many inflammatory mediators including IL-1 $\beta$ and IL- $6^{[4]}$.
\end{abstract}

Phytic acid (PA), also called inositol hexaphosphate, is a rich inositol substance in plants. It is also widely distributed in animal tissues and cells ${ }^{[5]}$. Studies have found that phytic acid has a number of physiological functions, including antioxidant, improve the body's immunity and anti-inflammatory effects, etc ${ }^{[6,7]}$. In this study, we used lipopolysaccharide (LPS) to activate microglia for establishing inflammatory model of neurodegenerative diseases to investigate the neuroprotection of phytic acid in parkinson's disease ${ }^{[8,9]}$.

\begin{abstract}
Materials and Methods
Material source

The BV-2 cell line was provided by Kunming animal of the Chinese Academy of Sciences. The cells were cultured in the DMEM medium of $10 \%$ fetal bovine serum. In the cell incubator of $37{ }^{\circ} \mathrm{C}$ and $5 \%$ carbon dioxide, the cells grew monolayer and were subcultured at about $80 \%$.
\end{abstract}

\section{Cell culture}

BV-2 microglia were cultured in high glucose DMEM 
medium containing $10 \%$ fetal bovine serum FBS and $1 \%$ double antibody, inoculated in culture bottle and incubated in $5 \% \mathrm{CO}_{2}, 37^{\circ} \mathrm{C}$ cell incubator. The cell monolayer adheres to the wall and changes the liquid in time. When the cells are covered with about $70 \%-80 \%$ of the culture bottle bottom, the cells are digested with $0.25 \%$ trypsin (containing $0.25 \%$ EDTA) and then subcultured for an average of 2-3 days. The cells in logarithmic growth phase were cultured in 6-well plate and 96-well plate respectively.

\section{MTT cell survival assay}

The BV-2 microglia in logarithmic growth period were inoculated in $96-$ well plate, $100 \mu \mathrm{L}$ per well. After 24 hours of adherent culture, the cells were replaced with fresh serum-free medium and treated with phytic acid. The concentration of phytic acid was set to $50 \mu \mathrm{mol} / \mathrm{L}, 100 \mu \mathrm{mol} / \mathrm{L}, 200 \mu \mathrm{mol} / \mathrm{L}$ alone or with LPS, the concentration of lipopolysaccharide was set to $10-1000 \mathrm{ng} / \mathrm{ml}$, and the blank control group $(0 \mu \mathrm{mol} / \mathrm{L})$. After 48 hours of culture, MTT solution was added to the cell solution $(10 \mu \mathrm{L})$, per pore was incubated at $37^{\circ} \mathrm{C}$ for $3 \mathrm{~h}$, then the culture medium was sucked out, then the optical density was measured at $10 \mathrm{~min}, 492 \mathrm{~nm}$ wavelength of $100 \mu \mathrm{L}$ dimethyl sulfoxide oscillation per well. Calculate cell survival rate.

\section{NO detection}

Detection of nitrite levels in cells by Griess assay. The microglia of logarithmic growth period were inoculated in 96 -well plate, $100 \mu \mathrm{L}$ per hole. After 24 hours of adherent culture, the cells were replaced with fresh serum-free medium and treated with phytic acid. After 48 hours of culture, the supernatant was removed and transferred to the 96-well cell culture plate, and the optical density was measured at the wavelength of 540 $\mathrm{nm}$ at room temperature with $50 \mu \mathrm{L}$ Griess reagent.

\section{ELISA detection}

The cells were cultured in 24-well plate and cultured to $80 \%$ confluence of cells. After 6 hours of culture, LPS (final concentration of $250 \mathrm{ng} / \mathrm{ml}$ ) was added to the culture medium containing the specified concentration of phytic acid. After 24 hours of culture, the culture medium of each pore was collected. Follow ELISA kit instructions.

\section{DAPI staining}

The BV-2 microglia in logarithmic growth period were inoculated in 6-well plate, and the cells ranged from 50 to $80 \%$ of the cells were treated with phytic acid and LPS at full time. After 24 hours of culture, the culture medium was removed, then washed with PBS, fixed with methanol solution for $5 \mathrm{~min}$, then washed with PBS, then added to the DAPI staining solution of 500ul, then stored at room temperature for $5 \mathrm{~min}$, then the dye solution was discarded. Wash with PBS twice and take pictures under fluorescence microscope.

\section{Statistical analysis}

The data were analyzed by SPSS 13.0 software. The results were consistent with the normal distribution, and single factor ANOVA was used to compare the results between groups and within groups.

\section{RESULTS}

Morphological changes of microglia induced by LPS.

As shown in Fig1, after treated by LPS, activated microglia exhibited different cellular shapes. The cell body became round and larger, less branches was shown, and length of branches became shorter. And the morphological changes were obvious.
A

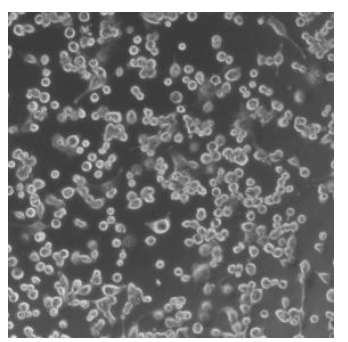

B

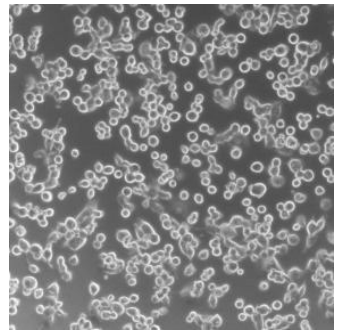

Fig1. A: Resting cell morphology $(\times 200)$; B: Activated cell morphology $(\times 200)$

\section{Effect of LPS on cell viability of microglia cells} Cell viability of $\mathrm{BV}-2$ was detected by 3-(4,5-Dimethythiazol-2-yl)-2,5-diphenyltetrazolium bromide (MTT) assay. Microglial neurons were incubated with different doses of LPS from $10 \mathrm{ng} / \mathrm{ml}$ to $1000 \mathrm{ng} / \mathrm{ml}$ for 24 hours, then the number of neuron was measured by MTT reagent. Following LPS's activation, cellular morphological changes were obviously seen under microscope. But the number of neurons had not significant differences.

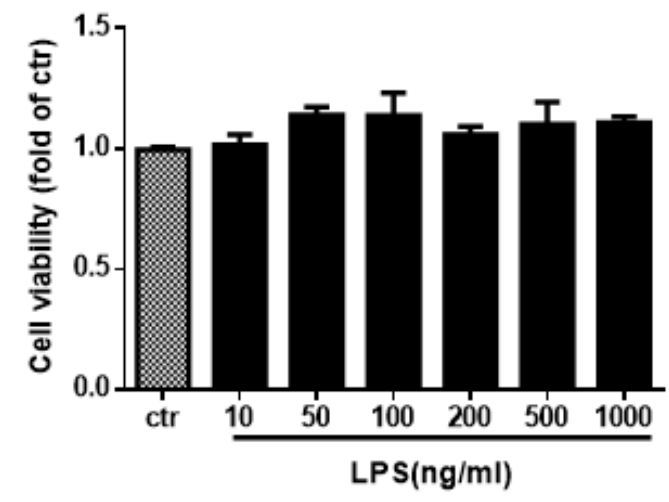

Fig2. Cytotoxicity effects of LPS on BV-2 cells

\section{Toxic effect of phytic acid on microglia}

In order to avoid toxic doses of phytic acid induced on microglia, MTT assay was also applied to assess cell viability of neurons exposed to different doses of phytic acid.As shown in Fig3, the result suggested there were not significantly different values among groups, which indicated little toxic effect of phytic acid on microglia. 


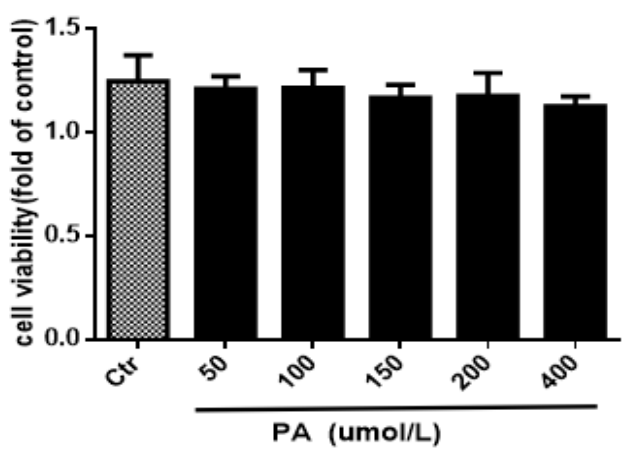

Fig3. Cytotoxicity effects of PA on BV-2 cells

Effects of phytic acid on NO release from LPS activated microglia cells

$\mathrm{NO}$ is one of the inflammatory markers produced by the activation of glial cells and is also an important indicator of neuroinflammation. Results in Fig4 indicated that NO release elevated after $200 \mathrm{ng} / \mathrm{ml}$ of LPS exposed to microglial neurons. While, pretreated with different concentrations of phytic acid for 24 hours, elevation of NO production induced by LPS attenuated in a dose-dependent manner.

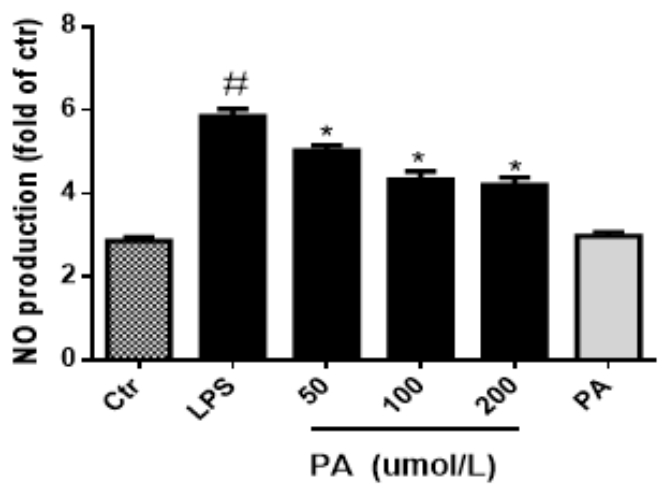

Fig4. Effect of PA on LPS-induced NO production in BV-2 cells

$\# \mathrm{P}<0.05$ vs control group; $* \mathrm{P}<0.05$ vs LPS treatment group

Effects of phytic acid on the release of microglial inflammatory factor IL-6 activated by LPS

Microglia participate in the inflammatory reaction of neurodegenerative diseases. With LPS stimulation, by releasing and secreting a series of neurotoxic substances and inflammatory factors such as IL-6, secondary brain tissue damage is caused.The effect of phytic acid on the expression of inflammatory factors was detected by ELISA. The results showed that the expression of IL-6 was upregulated after stimulation of LPS, and phytic acid could inhibit the expression of IL-6 in a dose-dependent manner.

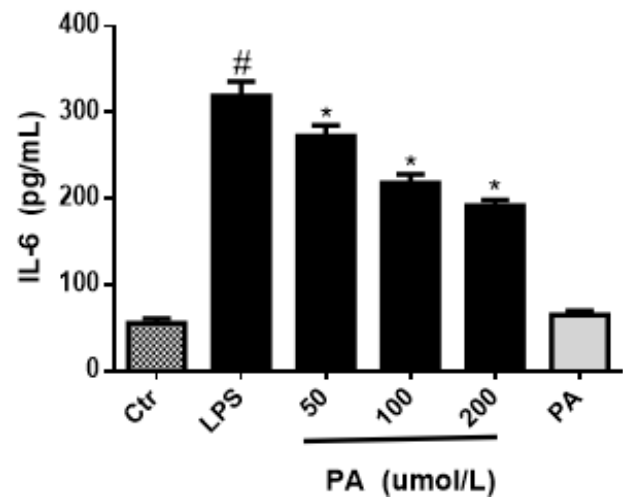

Fig5. Effect of PA on LPS-induced IL-6 production in $\mathrm{BV}-2$ cells

\#P<0.05 vs control group; $* \mathrm{P}<0.05$ vs LPS treatment group

Effect of phytic acid on morphological changes of apoptotic cells by DAPI staining

With DAPI staining, results in Fig6 showed the nucleus of the control group was intact and uniform, and the shape was regular. In the phytic acid group, most of the cells were chromatin inhomogeneous, chromatin condensed, agglutinated, nucleus cracked, and some nuclei were broken down.

A

B

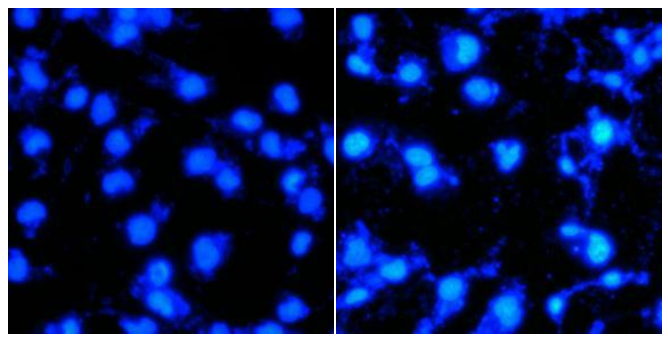

Fig6. A: Morphological changes of cells without phytic acid $(\times 400)$; B: Morphological changes of cells with phytic acid $(\times 400)$

\section{Discussion}

As the main immune cells in the brain, microglia function as nutritive neurons and immune surveillance. When the brain is injured or infected, microglia are activated by stimulation (such as the action of endogenous chemical LPS), which mainly play the role of phagocytosis, antigen presentation and release of inflammatory mediators (such as IL-6) ${ }^{[10]}$. Neuroinflammation caused by excessive activation of microglia is the most direct cause of synaptic dysfunction and cognitive decline of neurons ${ }^{[11]}$. It can be said that excessive neuroinflammation is the central link in the occurrence and development of neurodegenerative diseases. Therefore, neuroinflammation as a target, by inhibiting the excessive activation of microglia and promoting the release of inflammatory cytokines, can effectively prevent the deterioration of the disease ${ }^{[12]}$. Because phytic acid has strong neuroprotective and 
anti-inflammatory activity ${ }^{[13,14]}$, we established a neuroinflammatory model by culturing microglia in vitro to study the inhibitory effect of phytic acid on the inflammatory response induced by LPS.

The results of MTT test showed that there was no significant change in the survival rate of microglia after the treatment of phytic acid with LPS. Griess results showed that phytic acid could significantly inhibit NO release from BV-2 microglia activated by LPS.

Long-term sustained activation of microglia can secrete neurotoxic substances and inflammatory mediators, such as IL-6, TNF- $\alpha, \mathrm{NO}$, etc ${ }^{[15,16]}$. The results showed that the expression of IL-6, NO was up-regulated after LPS stimulation, and phytic acid inhibited the expression of IL-6, NO in a concentration-dependent manner. In this experiment, phytic acid was applied to the over-activated microglia. By DAPI staining, the morphological changes of typical apoptotic cells were observed by fluorescence microscope, which indicated that phytic acid could induce apoptosis of activated microglia.

To sum up, phytic acid can inhibit the excessive activation of BV-2 microglia stimulated by LPS, reduce the release of inflammatory factors, promote the apoptosis of over-activated microglia, and thus have obvious anti-inflammatory effect. It also indicates that phytic acid may have potential application value in central nervous degenerative diseases associated with inflammation. However, the more extensive role and the further action mechanism need to be further studied.

\section{REFERENCES}

1. Sugama S, Takenouchi T, Cho B P, et al.Possible roles of microglial cells for neurotoxicity in clinical neurodegenerative diseases and experimental animal models[J].Inflamm Allergy Drug Targets, 2009, 8( 4) : 277-84.

2. Dragone T, Cianciulli A, Calvello, et al. Resveratrol counteracts lipopolysaccharide-mediated microglial inflammation by modulating a SOCS-1 dependent signaling pathway[J]. Toxicol In Vitro, 2014, 28( 6) : 1126-35.
3. Choi D K, Koppula S, Suk K. Inhibitors of microglial neurotoxicity: focus on natural products[J]. Molecules,2011,16(2) : 1021-43.

4. Vizuete ML, Merino M, Venero JL, et al. Histamine infusion induces a selective dopaminergic neuronal deathalong with an inflammatory reaction in rat substantia nigra[J]. J Neurochemisty, 2000, 75( 2) :540-552 .

5. NRC. Nutrient requirements of poultry(9th ED)[M].1994.National Academy Press, Washington.D.C.

6. Gu M, Roy S, Raina K, et al. Inositol Hexaphosphate Suppresses Growth and Induces Apoptosis in Prostate Carcinoma Cells in Culture and Nude Mouse Xenograft: PI3K-Akt Pathway as Potential Target[J]. CANCER RESEA RCH, 2009,69(24):9465-9472.

7. Xu Q, Kanthasamy A G, Reddy M B. Neuroprotective effect of the natural iron chelator, phytic acid in a cell culture model of Parkinson's disease[J]. To xicology, 2008,245(1 -2):101-108.

8. Espinosa-Oliva A M, de Pablos R M, Herrera A J. Intracranial injection of LPS in rat as animal model of neuroinflammation[J]. Methods Mol Biol, 2013, 1041: 295 305 .

9. Huang F, Deng H M, Zhu M M, et al. Inhibitory effect of ferulic acid on inflammatory response in microglia induced by lipopolysaccharides[J]. Dongwuxue Yanjiu, 2011, 32( 3) : 311 -6 .

10. Wang Junhui, Sun Fengbo, Qin Luye, et al. Characteristics and functions of abnormally activated microglia $[\mathrm{J}]$. Advances in Physiology, 2008, 39 (1): 41.

11. Jung H Y, Nam K N, Woo B C, et al. Hirsutine, an indole alkaloid of Uncaria rhynchophylla, inhibits inflammation-mediated neurotoxicity and microglial activation[J]. Mol Med Rep, 2013, 7 ( 1) : 154 - 8.

12. Han L, Yin K, Zhang S, et al. Dalesconols $B$ inhibits lipopolysaccharide induced inflammation and suppresses $\mathrm{NF}-\mathrm{\kappa B}$ and p38/JNK activation in microglial cells $[\mathrm{J}]$ Neurochem Int, 2013,62 ( 7) : 913 - 21.

13. Zhang Z, Hou L, Li X, et al. Neuroprotection of inositol hexaphosphate and changes of mitochondrion mediated apoptotic pathway and alpha-synuclein aggregation in 6-OHDA induced parkinson's diseas cell model[J]. BRA IN RESEA RCH, 2016,1633:87-95.

14. Mythri R B, Harish G, Bharath M M. Therapeutic potential of natural products in Parkinson's disease[J]. Recent patents on endocrine, metabolic \& immune drug discovery, 2012,6(3):181-200

15. Lim J Y, Hwang B Y, Hwang $\mathrm{K}$ W, et al. Methylalpinumisoflavone inhibits lipopolysaccharide-induced inflammation in microglial cells by the NF-kappaB and MAPK signaling pathway[J]. Phytother Res, 2012, 26(12) : 1948- 56.

16. Wang Chao, Zhang Duwei, Xu Chong Neuroinflammation and Neurodegenerative Diseases Exploration $[\mathrm{J}]$. Electronic Journal of Clinical Medicine, 2017 (4): 65565. 